\title{
An application of interval-valued neutrosophic soft graphs in a decision making problem
}

\author{
Yildiray Celik and Guven Kara \\ Department of Mathematics, Ordu University, Ordu, Turkey \\ Received: 6 September 2018, Accepted: 6 October 2018 \\ Published online: 1 November 2018.
}

\begin{abstract}
In this paper, we introduce the concept of interval-valued neutrosophic soft graphs and gave some new operations such as parametric $\wedge$-intersection and parametric $\vee$ - union on interval-valued neutrosophic soft graphs. We have also applied the concept of interval-valued neutrosophic soft graph in a decision making problem and then gave an algorithm for the selection of optimal object.
\end{abstract}

Keywords: Interval-valued neutrosophic soft sets, interval-valued neutrosophic soft graphs, decision making problems.

\section{Introduction}

The concept of neutrosophic set which is a powerful mathematical tool for dealing with incomplete, indeterminate and inconsistent information was firstly discussed by Smarandache [1]. The neutrosophic sets are characterized by a truth-membership function $T$, an indeterminacy-membership function $I$ and a falsity membership function $F$ independently, which are within the real standard or nonstandard unit interval $]^{-} 0,1^{+}[$. Wang et al. [2] introduced the concept of interval-valued neutrosophic sets. Some works on neutrosophic sets, interval valued neutrosophic sets and their applications may be found on $[3,4,5,6,7,8]$.

The concept of soft set theory was initiated by Molodtsov [9]. It has been showed that soft sets have potential applications in different fields. The definition of neutrosophic soft sets was firstly given by Maji [10]. He also discussed many operations such as union, intersection and complement on neutrosophic soft sets. The concept of interval-valued neutrosophic soft set which is a generalization of neutrosophic soft sets was given by Deli [11].

Graph theory was firstly introduced by Euler [12]. Since then graph theory has become the most important part of combinatorial mathematics. A graph is used to create a relationship between a given set of elements. Each element can be represented by a vertex and the relationship between them can be represented by an edge. The concept of graph has been applied to different algebraic structures in mathematics.

In this paper, we define some new operations such as parametric $\wedge$-intersection and parametric $\vee-$ union on interval-valued neutrosophic soft graphs. We also present an application of interval-valued neutrosophic soft graph in a decision making problem and give an algorithm for the selection of optimal object based on given sets of information.

\section{Preliminaries}

Definition 1. [1] A neutrosophic set $A$ on the universe of discourse $U$ is defined as $A=\left\{\left\langle x, T_{A}(x), I_{A}(x)\right.\right.$, $\left.\left.F_{A}(x)\right\rangle, x \in U\right\}$, where the functions $T, I, F: U \rightarrow[0,1]$ define a truth-membership function, an indeterminacy-membership 
function and a falsity-membership function of an element $x \in U$ for a set $A$, respectively, with the condition ${ }^{-} 0 \leq T_{A}(x)+$ $I_{A}(x)+F_{A}(x) \leq 3^{+}$. The family of all neutrosphic sets on $U$ is denoted by $\mathscr{N}(U)$.

Definition 2. [10] Let $U$ be an inital universe set, $E$ be a set of parameters and $A \subseteq E$. If $F$ is a mapping given by $F: A \rightarrow \mathscr{N}(U)$, then a pair $(F, A)$ is called a neutrosophic soft set over $U$.

Definition 3. [11] Let $X$ be a space of objects with a generic element in X denoted by $x$. An interval-valued neutrosophic set (IVN-sets) $A$ in $X$ is characterized by truth-membership function $T_{A}$, an indeterminacy-membership function $I_{A}$ and a falsity-membership function $F_{A}$. For each point $x \in X, T_{A}, I_{A}$ and $F_{A} \subseteq[0,1]$. For this reason, we can represent each membership function for $x \in X$ as follows:

$$
T_{A}(x)=\left[\inf _{A}(x), \sup _{A}(x)\right], I_{A}(x)=\left[\inf _{A}(x), \operatorname{supI}_{A}(x)\right] \text { and } F_{A}(x)=\left[\inf F_{A}(x), \sup _{A}(x)\right]
$$

Thus, an IVN-set over $X$ can be represented by

$$
A=\left\{\left\langle x,\left[\inf _{A}(x), \sup _{A}(x)\right],\left[\operatorname{infI}_{A}(x), \operatorname{supI}_{A}(x)\right],\left[\inf _{A}(x), \operatorname{supF}_{A}(x)\right]\right\rangle \mid x \in X\right\}
$$

Definition 4. [11] Let $X$ be an initial universe set, IVN $(X)$ denotes the set of all interval-valued neutrosophic sets of $X$ and $E$ be a set of parameters that are describe the elements of $X$. An interval valued neutrosophic soft set $(\Upsilon, E)$ over $X$ is a set defined by a set valued function $\Upsilon$ representing a mapping $\Upsilon: E \rightarrow I V N(X)$. It can be written a set of ordered pairs $(\Upsilon, E)=\{(e, \Upsilon(e)): e \in E\}$. Here $\Upsilon$ is called approximate functions of the $(\Upsilon, E)$ and $\Upsilon(e)$ is called e-approximate value of $e \in E$.

We denote the set of all interval-valued neutrosophic soft sets over X by IVNS $(X)$.

Definition 5. [12] A graph $G^{*}$ consists of set of finite objects $V=\left\{v_{1}, v_{2}, v_{3}, \ldots, v_{n}\right\}$ called vertices and other set $E=\left\{e_{1}, e_{2}, e_{3}, \ldots, e_{n}\right\}$ whose elements are called edges. Usually a graph is denoted as $G^{*}=(V, E)$.

Definition 6. [13] An interval-valued neutrosophic graph of a graph $G^{*}=(V, E)$ is given by a pair $G=(A, B)$, where $A=<\left[T_{A}^{-}, T_{A}^{+}\right],\left[I_{A}^{-}, I_{A}^{+}\right],\left[F_{A}^{-}, F_{A}^{+}\right]>$is an interval-valued neutrosophic set on $V$ and $B=<\left[T_{B}^{-}, T_{B}^{+}\right],\left[I_{B}^{-}, I_{B}^{+}\right],\left[F_{B}^{-}, F_{A}^{+}\right]>$ is an interval-valued neutrosophic relation on $E$ such that

$$
\begin{array}{ll}
T_{B}^{-}\left(v_{i}, v_{j}\right) \leq \min \left\{T_{A}^{-}\left(v_{i}\right), T_{A}^{-}\left(v_{j}\right)\right\} & T_{B}^{+}\left(v_{i}, v_{j}\right) \leq \min \left\{T_{A}^{+}\left(v_{i}\right), T_{A}^{+}\left(v_{j}\right)\right\} \\
I_{B}^{-}\left(v_{i}, v_{j}\right) \geq \max \left\{I_{A}^{-}\left(v_{i}\right), I_{A}^{-}\left(v_{j}\right)\right\} & I_{B}^{+}\left(v_{i}, v_{j}\right) \geq \max \left\{I_{A}^{+}\left(v_{i}\right), I_{A}^{-}\left(v_{j}\right)\right\} \\
F_{B}^{-}\left(v_{i}, v_{j}\right) \geq \max \left\{F_{A}^{-}\left(v_{i}\right), F_{A}^{-}\left(v_{j}\right)\right\} & F_{B}^{+}\left(v_{i}, v_{j}\right) \geq \max \left\{F_{A}^{+}\left(v_{i}\right), F_{A}^{+}\left(v_{j}\right)\right\}
\end{array}
$$

for all $\left(v_{i}, v_{j}\right) \in E$.

Definition 7. [14] An interval-valued neutrosophic soft graph $\tilde{G}=\left(G^{*}, K, M, A\right)$ is a 4-tuple such that

(i) $G^{*}=(V, E)$ is a simple graph,

(ii) A is a non-empty set of parameters,

(iii) $(K, A)$ is an interval-valued neutrosophic soft set over $V$,

(iv) $(M, A)$ is an interval-valued neutrosophic soft set over $E$,

(v) $(K(e), M(e))$ is an an interval-valued neutrosophic soft subgraph of $G^{*}$ for all $e \in A$. That is,

$$
\begin{array}{ll}
\inf T_{M(e)}(x y) \leq \min \left\{\inf _{K(e)}(x), \inf T_{K(e)}(y)\right\} & \sup T_{M(e)}(x y) \leq \min \left\{\sup _{K(e)}(x), \sup _{K(e)}(y)\right\} \\
\inf I_{M(e)}(x y) \geq \max \left\{\operatorname{infI}_{K(e)}(x), \inf I_{K(e)}(y)\right\} & \operatorname{supI}_{M(e)}(x y) \geq \max \left\{\operatorname{supI}_{K(e)}(x), \operatorname{supI}_{K(e)}(y)\right\} \\
\inf F_{M(e)}(x y) \geq \max \left\{\inf _{K(e)}(x), \inf F_{K(e)}(y)\right\} & \operatorname{supF}_{M(e)}(x y) \geq \max \left\{\sup _{K(e)}(x), \operatorname{supF}_{K(e)}(y)\right\}
\end{array}
$$

The interval-valued neutrosophic graph $(K(e), M(e))$ is denoted by $H(e)$ for convenience. An interval-valued neutrosophic soft graph is a parametrized family of interval-valued neutrosophic graphs. The set of all interval-valued neutrosophic soft graphs of $G^{*}$ is denoted by $\operatorname{IVN}\left(G^{*}\right)$. 
Example 1. Consider a simple graph $G^{*}=(V, E)$ such that $V=\left\{v_{1}, v_{2}, v_{3}\right\}$ and $E=\left\{v_{1} v_{2}, v_{2} v_{3}, v_{1} v_{3}\right\}$. Let $A=\left\{e_{1}, e_{2}\right\}$ be set of parameter and let $(K, A)$ be an interval-valued neutrosophic soft set over $V$ with its approximate function $K$ : $A \rightarrow I V N(V)$ defined by

$$
\begin{aligned}
& K\left(e_{1}\right)=\left\{\left\langle v_{1},[0.5,0.6],[0.3,0.4],[0.2,0.3]\right\rangle,\left\langle v_{2},[0.4,0.5],[0.3,0.4],[0.6,0.7]\right\rangle,\left\langle v_{3},[0.6,0.7],[0.2,0.3],[0.1,0.2]\right\rangle\right\} \\
& K\left(e_{2}\right)=\left\{\left\langle v_{1},[0.3,0.5],[0.7,0.8],[0.4,0.5]\right\rangle,\left\langle v_{2},[0.2,0.3],[0.4,0.5],[0.4,0.5]\right\rangle,\left\langle v_{3},[0.2,0.4],[0.3,0.5],[0.1,0.2]\right\rangle\right\}
\end{aligned}
$$

Let $(M, A)$ be an interval-valued neutrosophic soft set over $E$ with its approximate function $M: E \rightarrow I V N(E)$ defined by

$M\left(e_{1}\right)=\left\{\left\langle v_{1} v_{2},[0.3,0.4],[0.4,0.5],[0.7,0.8]\right\rangle,\left\langle v_{2} v_{3},[0.1,0.2],[0.5,0.6],[0.6,0.8]\right\rangle,\left\langle v_{3} v_{1},[0.2,0.3],[0.3,0.6],[0.4,0.5]\right\rangle\right\}$, $M\left(e_{2}\right)=\left\{\left\langle v_{1} v_{2},[0.2,0.3],[0.7,0.8],[0.5,0.6]\right\rangle,\left\langle v_{2} v_{3},[0.1,0.2],[0.6,0.7],[0.5,0.6]\right\rangle,\left\langle v_{3} v_{1},[0.1,0.4],[0.8,0.9],[0.6,0.7]\right\rangle\right\}$.

Clearly, $H\left(e_{1}\right)=\left(K\left(e_{1}\right), M\left(e_{1}\right)\right)$ and $H\left(e_{2}\right)=\left(K\left(e_{2}\right), M\left(e_{2}\right)\right)$ are interval-valued neutrosophic graphs corresponding to the paramteters $e_{1}$ and $e_{2}$ as shown in figure below, respectively.

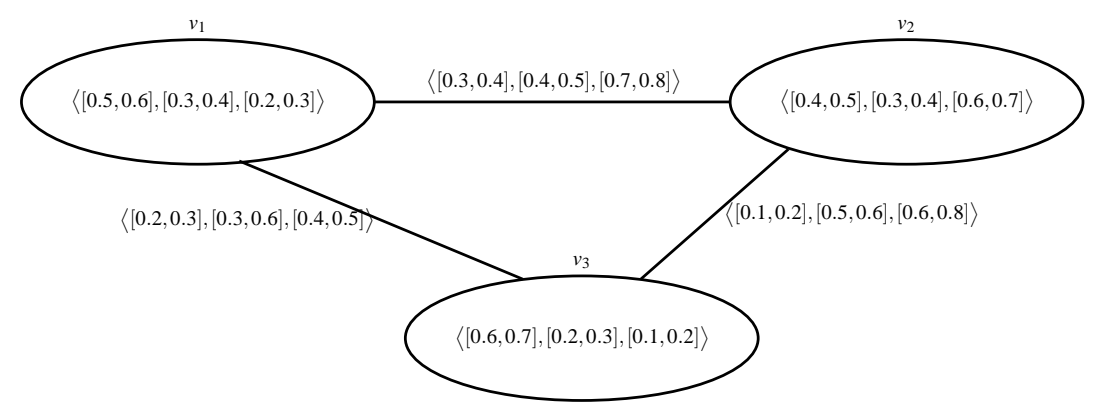

Fig. 1: $H\left(e_{1}\right)$ interval valued neutrosophic graph

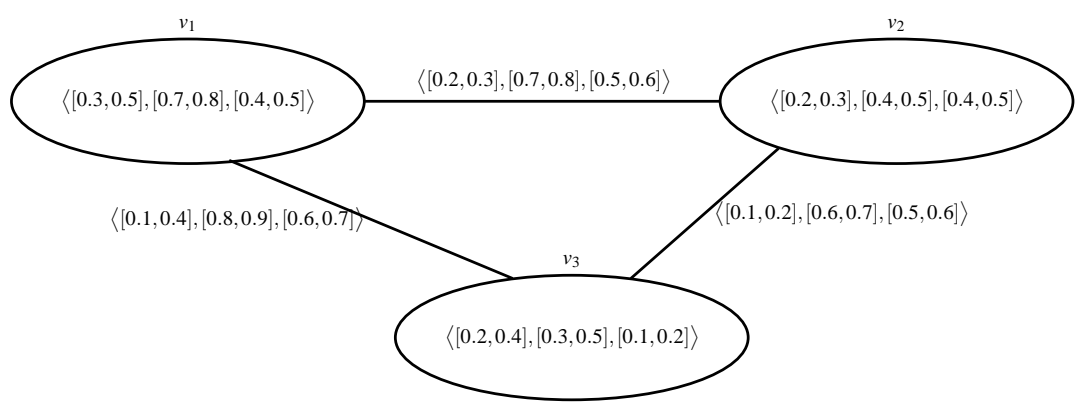

Fig. 2: $H\left(e_{2}\right)$ interval valued neutrosophic graph

Hence $\tilde{G}=\left\{H\left(e_{1}\right), H\left(e_{2}\right)\right\}$ is an interval-valued neutrosophic soft graph of $G^{*}$. Tabular representation of an intervalvalued neutrosophic soft graph $\tilde{G}$ is given in Table 1 . 
Table 1: Interval-valued neutrosophic soft graph $\tilde{G}$

\begin{tabular}{|c|c|c|c|}
\hline$K$ & $v_{1}$ & $v_{2}$ & $v_{3}$ \\
\hline$e_{1}$ & $\langle[0.5,0.6],[0.3,0.4],[0.2,0.3]\rangle$ & $\langle[0.4,0.5],[0.3,0.4],[0.6,0.7]\rangle$ & $\langle[0.6,0.7],[0.2,0.3],[0.1,0.2]\rangle$ \\
$e_{2}$ & $\langle[0.3,0.5],[0.7,0.8],[0.4,0.5]\rangle$ & $\langle[0.2,0.3],[0.4,0.5],[0.4,0.5]\rangle$ & $\langle[0.2,0.4],[0.3,0.5],[0.1,0.2]\rangle$ \\
\hline \hline$M$ & $v_{1} v_{2}$ & $v_{2} v_{3}$ & $v_{3} v_{1}$ \\
\hline$e_{1}$ & $\langle[0.3,0.4],[0.4,0.5],[0.7,0.8]\rangle$ & $\langle[0.1,0.2],[0.5,0.6],[0.6,0.8]\rangle$ & $\langle[0.2,0.3],[0.3,0.6],[0.4,0.5]\rangle$ \\
$e_{2}$ & $\langle[0.2,0.3],[0.7,0.8],[0.5,0.6]\rangle$ & $\langle[0.1,0.2],[0.6,0.7],[0.5,0.6]\rangle$ & $\langle[0.1,0.4],[0.8,0.9],[0.6,0.7]\rangle$ \\
\hline
\end{tabular}

Definition 8. [14] Let $\tilde{G}_{1}=\left(G^{*}, K_{1}, M_{1}, A\right)$ and $\tilde{G}_{2}=\left(G^{*}, K_{2}, M_{2}, B\right)$ be two interval-valued neutrosophic soft graphs of $G^{*}=(V, E)$. Then $\tilde{G}_{1}$ is an interval valued neutrosophic soft subgraph of $\tilde{G}_{2}$ if

(i) $A \subseteq B$

(ii) $H_{1}(e)$ is a subgraph of $H_{2}(e)$ for all $e \in A$.

Definition 9. Let $\tilde{G}=\left(G^{*}, K, M, A\right)$ be an interval-valued neutrosophic soft graph of $G^{*}=(V, E)$ and $A=\left\{e_{1}, e_{2}, \ldots, e_{n}\right\}$ be a set of parameters. The parametric $\wedge$-intersection of subgraphs of $\tilde{G}$ is denoted by

$$
M(e)=M\left(e_{1}\right) \tilde{\wedge} M\left(e_{2}\right) \tilde{\wedge} \ldots \tilde{\wedge} M\left(e_{n}\right)
$$

where $e=e_{1} \wedge e_{2} \wedge \ldots \wedge e_{n}$ and for all $x y \in E$

$$
\begin{aligned}
& \inf _{M(e)}(x y)=\min \left\{\inf _{M\left(e_{1}\right)}(x y), \inf T_{M\left(e_{2}\right)}(x y), \ldots, \inf T_{M\left(e_{n}\right)}(x y)\right\} \\
& \sup _{M(e)}(x y)=\min \left\{\sup _{M\left(e_{1}\right)}(x y), \sup _{M\left(e_{2}\right)}(x y), \ldots, \sup _{M\left(e_{n}\right)}(x y)\right\} \\
& \operatorname{infI}_{M(e)}(x y)=\max \left\{\operatorname{infI}_{M\left(e_{1}\right)}(x y), \inf I_{M\left(e_{2}\right)}(x y), \ldots, \inf I_{M\left(e_{n}\right)}(x y)\right\} \\
& \operatorname{supI}_{M(e)}(x y)=\max \left\{\operatorname{supI}_{M\left(e_{1}\right)}(x y), \operatorname{supI}_{M\left(e_{2}\right)}(x y), \ldots, \operatorname{supI}_{M\left(e_{n}\right)}(x y)\right\} \\
& \operatorname{infF}_{M(e)}(x y)=\max \left\{\inf _{M\left(e_{1}\right)}(x y), \inf _{M\left(e_{2}\right)}(x y), \ldots, \operatorname{infF}_{M\left(e_{n}\right)}(x y)\right\} \\
& \operatorname{supF}_{M(e)}(x y)=\max \left\{\sup _{M\left(e_{1}\right)}(x y), \operatorname{supF}_{M\left(e_{2}\right)}(x y), \ldots, \operatorname{supF}_{M\left(e_{n}\right)}(x y)\right\} .
\end{aligned}
$$

Definition 10. Let $\tilde{G}=\left(G^{*}, K, M, A\right)$ be an interval-valued neutrosophic soft graph of $G^{*}=(V, E)$ and $A=\left\{e_{1}, e_{2}, \ldots, e_{n}\right\}$ be a set of parameters. The parametric $\vee$-union of subgraphs of $\tilde{G}$ is denoted by

$$
M(e)=M\left(e_{1}\right) \tilde{V} M\left(e_{2}\right) \tilde{V} \ldots \tilde{V} M\left(e_{n}\right)
$$

where $e=e_{1} \vee e_{2} \vee \ldots \vee e_{n}$ and for all $x y \in E$

$$
\begin{aligned}
& \inf _{M(e)}(x y)=\min \left\{\inf T_{M\left(e_{1}\right)}(x y), \inf T_{M\left(e_{2}\right)}(x y), \ldots, \inf T_{M\left(e_{n}\right)}(x y)\right\} \\
& \sup _{M(e)}(x y)=\min \left\{\sup T_{M\left(e_{1}\right)}(x y), \sup _{M\left(e_{2}\right)}(x y), \ldots, \sup _{M\left(e_{n}\right)}(x y)\right\} \\
& \operatorname{infI}_{M(e)}(x y)=\max \left\{\operatorname{infI}_{M\left(e_{1}\right)}(x y), \inf I_{M\left(e_{2}\right)}(x y), \ldots, \inf I_{M\left(e_{n}\right)}(x y)\right\} \\
& \operatorname{supI}_{M(e)}(x y)=\max \left\{\operatorname{supI}_{M\left(e_{1}\right)}(x y), \operatorname{supI}_{M\left(e_{2}\right)}(x y), \ldots, \operatorname{supI}_{M\left(e_{n}\right)}(x y)\right\} \\
& \inf _{M(e)}(x y)=\max \left\{\inf _{M\left(e_{1}\right)}(x y), \inf _{M\left(e_{2}\right)}(x y), \ldots, \inf F_{M\left(e_{n}\right)}(x y)\right\} \\
& \sup _{M(e)}(x y)=\max \left\{\sup _{M\left(e_{1}\right)}(x y), \operatorname{supF}_{M\left(e_{2}\right)}(x y), \ldots, \sup _{M\left(e_{n}\right)}(x y)\right\} .
\end{aligned}
$$

Example 2. Consider a simple graph $G^{*}=(V, E)$ such that $V=\left\{v_{1}, v_{2}, v_{3}, v_{4}, v_{5}\right\}$ and $E=\left\{v_{1} v_{2}, v_{1} v_{3}, v_{1} v_{4}, v_{1} v_{5}, v_{2} v_{3}, v_{2} v_{4}, v_{2} v_{5}, v_{3} v_{4}, v_{3} v_{5}, v_{4} v_{5}\right\}$.

Let $A=\left\{e_{1}, e_{2}, e_{3}\right\}$ be a set of parameters and consider interval-valued neutrosophic soft graph $\tilde{G}=\left(G^{*}, K, M, A\right)$ as follows. 


$$
\begin{aligned}
& \tilde{G}=\left\{H\left(e_{1}\right), H\left(e_{2}\right), H\left(e_{3}\right)\right\}=\left\{\left(K\left(e_{1}\right), M\left(e_{1}\right)\right),\left(K\left(e_{2}\right), M\left(e_{2}\right)\right),\left(K\left(e_{3}\right), M\left(e_{3}\right)\right)\right\} \\
& K\left(e_{1}\right)=\{\left\langle v_{1},[0.2,0.4],[0.3,0.5],[0.6,0.8]\right\rangle,\left\langle v_{2},[0.1,0.3],[0.4,0.7],[0.3,0.6]\right\rangle, \\
&\left\langle v_{3},[0.3,0.5],[0.2,0.3],[0.4,0.5]\right\rangle,\left\langle v_{4},[0.5,0.6],[0.5,0.8],[0.2,0.4]\right\rangle, \\
&\left.\left\langle v_{5},[0.4,0.7],[0.6,0.9],[0.7,0.9]\right\rangle\right\} \\
& M\left(e_{1}\right)=\left\langle\left\langle v_{1} v_{2},[0.1,0.3],[0.6,0.8],[0.7,0.8]\right\rangle, v_{1} v_{3},[0.2,0.4],[0.5,0.6],[0.8,0.9]\right\rangle, \\
&\left.\left\langle v_{1} v_{5},[0.1,0.2],[0.8,0.9],[0.7,0.9]\right\rangle, v_{2} v_{3},[0.1,0.3],[0.5,0.7],[0.4,0.6]\right\rangle, \\
&\left.\left\langle v_{2} v_{4},[0.1,0.2],[0.6,0.9],[0.5,0.7]\right\rangle, v_{2} v_{5},[0.1,0.3],[0.8,0.9],[0.7,0.9]\right\rangle, \\
&\left.\left.\left\langle v_{3} v_{4},[0.3,0.4],[0.7,0.9],[0.8,0.9]\right\rangle, v_{3} v_{5},[0.2,0.5],[0.8,0.9],[0.7,0.9]\right\rangle\right\} . \\
& K\left(e_{2}\right)=\left\{\left\langle v_{1},[0.2,0.3],[0.6,0.7],[0.2,0.3]\right\rangle,\left\langle v_{2},[0.2,0.4],[0.3,0.5],[0.3,0.4]\right\rangle,\right.\left\langle v_{3},[0.6,0.8],[0.3,0.4],[0.6,0.7]\right\rangle,\left\langle v_{4},[0.1,0.2],[0.5,0.6],[0.7,0.8]\right\rangle, \\
&\left.\left\langle v_{5},[0.3,0.4],[0.6,0.7],[0.8,0.9]\right\rangle\right\}, \\
& M\left(e_{2}\right)=\left\{\left\langle v_{1} v_{2},[0.2,0.3],[0.7,0.8],[0.5,0.7]\right\rangle,\left\langle v_{1} v_{4},[0.1,0.2],[0.8,0.9],[0.7,0.9]\right\rangle,\right.\left\langle v_{1} v_{5},[0.3,0.4],[0.7,0.9],[0.8,0.9]\right\rangle,\left\langle v_{2} v_{3},[0.1,0.3],[0.4,0.5],[0.7,0.8]\right\rangle, \\
&\left.\left\langle v_{2} v_{5},[0.2,0.4],[0.8,0.9],[0.8,0.9]\right\rangle,\left\langle v_{3} v_{4},[0.1,0.2],[0.6,0.7],[0.8,0.9]\right\rangle\right\} . \\
& K\left(e_{3}\right)=\left\{\left\langle v_{1},[0.3,0.5],[0.4,0.5],[0.5,0.6]\right\rangle,\left\langle v_{2},[0.2,0.4],[0.3,0.4],[0.6,0.7]\right\rangle,\right.\left\langle v_{3},[0.1,0.3],[0.5,0.7],[0.4,0.6]\right\rangle,\left\langle v_{4},[0.4,0.6],[0.2,0.3],[0.3,0.5]\right\rangle, \\
&\left.\left\langle v_{5},[0.3,0.4],[0.6,0.7],[0.7,0.8]\right\rangle\right\} \\
& M\left(e_{3}\right)=\left\{\left\langle v_{1} v_{2},[0.2,0.3],[0.5,0.6],[0.7,0.8]\right\rangle,\left\langle v_{1} v_{5},[0.3,0.4],[0.7,0.8],[0.8,0.9]\right\rangle,\right.\left\langle v_{2} v_{3},[0.1,0.2],[0.6,0.7],[0.7,0.9]\right\rangle,\left\langle v_{2} v_{5},[0.2,0.4],[0.8,0.9],[0.7,0.8]\right\rangle, \\
&\left\langle v_{3} v_{4},[0.1,0.3],[0.5,0.8],[0.6,0.7]\right\rangle,\left\langle v_{3} v_{5},[0.1,0.2],[0.7,0.8],[0.7,0.9]\right\rangle, \\
&\left.\left\langle v_{4} v_{5},[0.1,0.4],[0.6,0.7],[0.8,0.9]\right\rangle\right\} .
\end{aligned}
$$

Clearly, $H\left(e_{1}\right), H\left(e_{2}\right)$ and $H\left(e_{3}\right)$ are subgraphs of $\tilde{G}$. They are shown in matrix form in the below for $e_{1}, e_{2}$ and $e_{3}$ respectively.

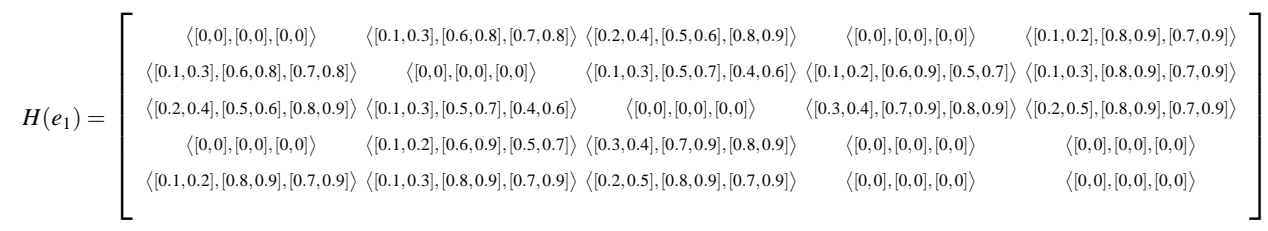

$$
\begin{aligned}
& H\left(e_{2}\right)=\left[\begin{array}{ccccc}
\langle[0,0],[0,0],[0,0]\rangle & \langle[0.2,0.3],[0.7,0.8],[0.5,0.7]\rangle & \langle[0,0],[0,0],[0,0]\rangle & \langle[0.1,0.2],[0.8,0.9],[0.7,0.9]\rangle & \langle[0.3,0.4],[0.7,0.9],[0.8,0.9]\rangle \\
\langle[0.2,0.3],[0.7,0.8],[0.5,0.7]\rangle & \langle[0,0],[0,0],[0,0]\rangle & \langle[0.1,0.3],[0.4,0.5],[0.7,0.8]\rangle & \langle[0,0],[0,0],[0,0]\rangle & \langle[0.2,0.4],[0.8,0.9],[0.8,0.9]\rangle \\
\langle[0,0],[0,0],[0,0]\rangle & \langle[0.1,0.3],[0.4,0.5],[0.7,0.8]\rangle & \langle[0,0],[0,0],[0,0]\rangle & \langle[0.1,0.2],[0.6,0.7],[0.8,0.9]\rangle & \langle[0,0],[0,0],[0,0]\rangle \\
\langle[0.1,0.2],[0.8,0.9],[0.7,0.9]\rangle & \langle[0,0],[0,0],[0,0]\rangle & \langle[0.1,0.2],[0.6,0.7],[0.8,0.9]\rangle & \langle[0,0],[0,0],[0,0]\rangle & \langle[0,0],[0,0],[0,0]\rangle \\
\langle[0.3,0.4],[0.7,0.9],[0.8,0.9]\rangle & \langle[0.2,0.4],[0.8,0.9],[0.8,0.9]\rangle & \langle[0,0],[0,0],[0,0]\rangle & \langle[0,0],[0,0],[0,0]\rangle & \langle[0,0],[0,0],[0,0]\rangle
\end{array}\right]
\end{aligned}
$$




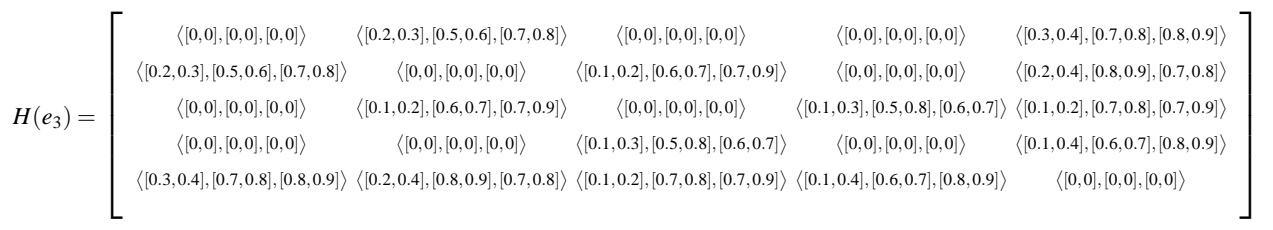

By using Definition 9, we obtain the parametric $\wedge$-intersection of subgraphs of $\tilde{G}$ as follows.

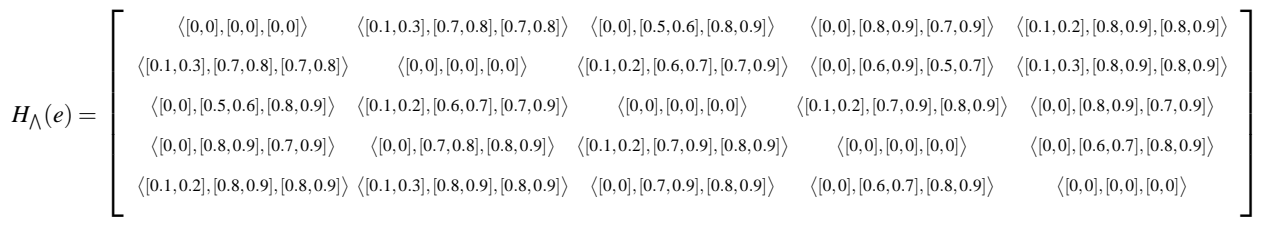

By using Definition 10, we obtain the parametric $\vee$-union of subgraphs of $\tilde{G}$ as follows.

$$
H_{\bigvee}(e)=\left[\begin{array}{ccccc}
\langle[0,0],[0,0],[0,0]\rangle & \langle[0.2,0.3],[0.5,0.6],[0.7,0.8]\rangle & \langle[0.2,0.4],[0,0],[0,0]\rangle & \langle[0.1,0.2],[0,0],[0,0]\rangle & \langle[0.3,0.04],[0.7,0.8],[0.8,0.9]\rangle \\
\langle[0.2,0.3],[0.5,0.6],[0.5,0.7]\rangle & \langle[0,0],[0,0],[0,0]\rangle & \langle[0.1,0.3],[0.4,0.5],[0.4,0.6]\rangle & \langle[0.1,0.2],[0,0],[0,0]\rangle & \langle[0.2,0.4],[0.8,0.9],[0.7,0.9]\rangle \\
\langle[0.2,0.4],[0,0],[0,0]\rangle & \langle[0.1,0.3],[0.4,0.5],[0.4,0.6]\rangle & \langle[0,0],[0,0],[0,0]\rangle & \langle[0.3,0.4],[0.5,0.7],[0.6,0.7]\rangle & \langle[0.2,0.5],[0,0],[0,0]\rangle \\
\langle[0.1,0.2],[0,0],[0,0]\rangle & \langle[0.2,0.3],[0,0],[0,0]\rangle & \langle[0.3,0.4],[0.5,0.7],[0.6,0.7]\rangle & \langle[0,0],[0,0],[0,0]\rangle & \langle[0.1,0.4],[0,0],[0,0]\rangle \\
\langle[0.3,0.4],[0.7,0.0],[0.7,0.9]\rangle & \langle[0.2,0.04],[0.8,0.9],[0.7,0.8]\rangle & \langle[0.2,0.5],[0,0],[0,0]\rangle & \langle[0.1,0.4],[0,0],[0,0]\rangle & \langle[0,0],[0,0],[0,0]\rangle
\end{array}\right]
$$

\section{An Application of Interval-valued Neutrosophic Soft Graphs in a Decision Making Problem}

Interval-valued neutrosophic soft set has various applications in decision making problems and used to deal with uncertainties from our real life problems. In this section, we applied the concept of interval-valued neutrosophic soft sets in a decision making problem and then gave an algorithm for the selection of optimal object based on given sets of information. Suppose that $V=\left\{t_{1}, t_{2}, t_{3}, t_{4}, t_{5}\right\}$ be the set of five football clubs under consideration which an investor is going to purchase a football club on the basis of wishing parameters or attributes set $A=\left\{e_{1}=\right.$ performance,$e_{2}=$ status,$e_{3}=$ squad value $\}$.

Let $(K, A)$ be an interval-valued neutrosophic soft graphs on $V$ which describe the value of the football clubs based upon the given parameters $e_{1}=$ performance, $e_{2}=$ status, $e_{3}=$ squad value, respectively, as given by

$$
\begin{aligned}
K\left(e_{1}\right)=\left\{\left\langle t_{1},[0.1,0.2],[0.5,0.6],[0.3,0.5]\right\rangle,\left\langle t_{2},[0.2,0.5],[0.4,0.7],[0.5,0.8]\right\rangle,\right. & \left\langle t_{3},[0.5,0.6],[0.6,0.8],[0.1,0.3]\right\rangle,\left\langle t_{4},[0.3,0.4],[0.2,0.3],[0.1,0.3]\right\rangle, \\
& \left.\left\langle t_{5},[0.5,0.6],[0.7,0.8],[0.1,0.3]\right\rangle\right\} \\
K\left(e_{2}\right)=\left\{\left\langle t_{1},[0.3,0.4],[0.4,0.7],[0.1,0.2]\right\rangle,\left\langle t_{2},[0.3,0.5],[0.2,0.3],[0.1,0.2]\right\rangle,\right. & \left\langle t_{3},[0.7,0.8],[0.5,0.6],[0.4,0.5]\right\rangle,\left\langle t_{4},[0.2,0.4],[0.1,0.2],[0.8,0.9]\right\rangle, \\
& \left.\left\langle t_{5},[0.4,0.5],[0.3,0.4],[0.6,0.7]\right\rangle\right\} \\
K\left(e_{3}\right)=\left\{\left\langle t_{1},[0.2,0.3],[0.3,0.5],[0.2,0.4]\right\rangle,\left\langle t_{2},[0.4,0.5],[0.3,0.4],[0.5,0.7]\right\rangle,\right. & \left\langle t_{3},[0.6,0.7],[0.4,0.5],[0.3,0.4]\right\rangle,\left\langle t_{4},[0.3,0.4],[0.2,0.3],[0.6,0.7]\right\rangle, \\
& \left.\left\langle t_{5},[0.1,0.2],[0.4,0.5],[0.5,0.6]\right\rangle\right\} .
\end{aligned}
$$


Let $(M, A)$ be an interval-valued neutrosophic soft set on $E=\left\{t_{1} t_{2}, t_{1} t_{3}, t_{1} t_{4}, t_{1} t_{5}, t_{2} t_{3}, t_{2} t_{4}, t_{2} t_{5}\right.$, $\left.t_{3} t_{4}, t_{3} t_{5}, t_{4} t_{5}\right\}$ which describe the value obtained by comparing two football clubs according to each parameter as given by

$$
\begin{aligned}
M\left(e_{1}\right)=\left\{\left\langle t_{1} t_{2},[0.1,0.2],[0.6,0.8],[0.6,0.9]\right\rangle, t_{2} t_{3},[0.1,0.4],[0.8,0.9],[0.6,0.9]\right\rangle, & \left.\left\langle t_{1} t_{5},[0.1,0.2],[0.8,0.9],[0.4,0.6]\right\rangle, t_{2} t_{4},[0.2,0.3],[0.7,0.8],[0.7,0.9]\right\rangle, \\
& \left.\left\langle t_{3} t_{4},[0.2,0.3],[0.7,0.8],[0.2,0.4]\right\rangle, t_{2} t_{5},[0.1,0.3],[0.8,0.9],[0.5,0.8]\right\rangle, \\
& \left.\left\langle t_{3} t_{5},[0.3,0.4],[0.7,0.9],[0.5,0.6]\right\rangle\right\}, \\
M\left(e_{2}\right)=\left\{\left\langle t_{1} t_{2},[0.2,0.3],[0.5,0.6],[0.3,0.4]\right\rangle,\left\langle t_{2} t_{3},[0.3,0.4],[0.6,0.7],[0.5,0.6]\right\rangle,\right. & \left\langle t_{1} t_{5},[0.1,0.2],[0.6,0.8],[0.8,0.9]\right\rangle,\left\langle t_{3} t_{4},[0.2,0.3],[0.6,0.7],[0.8,0.9]\right\rangle, \\
& \left.\left\langle t_{2} t_{5},[0.3,0.4],[0.4,0.5],[0.7,0.8]\right\rangle\right\} \\
M\left(e_{3}\right)=\left\{\left\langle t_{1} t_{2},[0.1,0.2],[0.4,0.6],[0.6,0.8]\right\rangle,\left\langle t_{2} t_{3},[0.3,0.4],[0.5,0.6],[0.7,0.8]\right\rangle,\right. & \left\langle t_{1} t_{5},[0.1,0.2],[0.5,0.6],[0.6,0.7]\right\rangle,\left\langle t_{3} t_{4},[0.1,0.2],[0.6,0.7],[0.6,0.8]\right\rangle, \\
& \left\langle t_{4} t_{5},[0.1,0.2],[0.5,0.6],[0.7,0.8]\right\rangle,\left\langle t_{2} t_{4},[0.2,0.3],[0.4,0.5],[0.8,0.9]\right\rangle, \\
& \left.\left\langle t_{2} t_{5},[0.1,0.2],[0.7,0.8],[0.8,0.9]\right\rangle\right\} .
\end{aligned}
$$

The interval-valued neutrosophic graphs $H\left(e_{1}\right)=\left(K\left(e_{1}\right), M\left(e_{1}\right)\right), H\left(e_{2}\right)=\left(K\left(e_{2}\right), M\left(e_{2}\right)\right)$ and $H\left(e_{3}\right)=\left(K\left(e_{3}\right), M\left(e_{3}\right)\right)$ corresponding to the parameters $e_{1}, e_{2}$ and $e_{3}$, respectively, are represented by the following incidence matrices.

$$
H\left(e_{1}\right)=\left[\begin{array}{ccccc}
\langle[0,0],[0,0],[0,0]\rangle & \langle[0.1,0.2],[0.6,0.8],[0.6,0.9]\rangle & \langle[0,0],[0,0],[0,0]\rangle & \langle[0,0],[0,0],[0,0]\rangle & \langle[0.1,0.2],[0.8,0.0 .9],[0.4,0.6]\rangle \\
\langle[0.1,0.2],[0.6,0.8],[0.6,0.9]\rangle & \langle[0,0],[0,0],[0,0]\rangle & \langle[0.1,0.4],[0.8,0.9],[0.6,0.9]\rangle & \langle[0.2,0.3],[0.7,0.8],[0.7,0.9]\rangle & \langle[0.1,0.3],[0.8,0.9],[0.5,0.8]\rangle \\
\langle[0,0],[0,0],[0,0]\rangle & \langle[0.1,0.4],[0.8,0.9],[0.6,0.9]\rangle & \langle[0,0],[0,0],[0,0]\rangle & \langle[0.2,0.3],[0.7,0.8],[0.2,0.4]\rangle & \langle[0.3,0.4],[0.7,0.9],[0.5,0.6]\rangle \\
\langle[0,0],[0,0],[0,0]\rangle & \langle[0.2,0.3],[0.7,0.8],[0.7,0.9]\rangle & \langle[0.2,0.3],[0.7,0.8],[0.2,0.4]\rangle & \langle[0,0],[0,0],[0,0]\rangle & \langle[0,0],[0,0],[0,0]\rangle \\
\langle[0.1,0.2],[0.8,0.9],[0.4,0.6]\rangle & \langle[0.1,0.3],[0.8,0.9],[0.5,0.8]\rangle & \langle[0.3,0.4],[0.7,0.9],[0.5,0.6]\rangle & \langle[0,0],[0,0],[0,0]\rangle & \langle[0,0],[0,0],[0,0]\rangle
\end{array}\right]
$$

$$
H\left(e_{2}\right)=\left[\begin{array}{ccccc}
\langle[0,0],[0,0],[00,0]\rangle & \langle[0.2,0.3],[0.5,0.6],[0.3,0.4]\rangle & \langle[0,0],[0,0],[0,0]\rangle & \langle[0,0],[0,0],[0,0]\rangle & \langle[0.1,0.2],[0.6,0.0 .,[0.8,0.9]\rangle \\
\langle[0.1,0.2],[0.6,0.8],[0.6,0.9]\rangle & \langle[0,0],[0,0],[0,0]\rangle & \langle[0.3,0.4],[0.6,0.7],[0.5,0.6]\rangle & \langle[0,0],[0,0],[0,0]\rangle & \langle[0.3,0.4],[0.4,0.5],[0.7,0.8]\rangle \\
\langle[0,0],[0,0],[0,0]\rangle & \langle[0.3,0.4],[0.6,0.7],[0.5,0.6]\rangle & \langle[0,0],[0,0],[0,0]\rangle & \langle[0.2,0.3],[0.6,0.7],[0.8,0.9]\rangle & \langle[0,0],[0,0],[0,0]\rangle \\
\langle[0,0],[0,0],[0,0]\rangle & \langle[0,0],[0,0],[0,0]\rangle & \langle[0.2,0.3],[0.6,0.7],[0.8,0.9]\rangle & \langle[0,0],[0,0],[0,0]\rangle & \langle[0,0],[0,0],[0,0]\rangle \\
\langle[0.1,0.2],[0.6,0.8],[0.8,0.9]\rangle] & \langle[0.3,0.4],[0.4,0.5],[0.7,0.8]\rangle & \langle[0,0],[0,0],[0,0]\rangle & \langle[0,0],[0,0],[0,0]\rangle & \langle[0,0],[0,0],[0,0]\rangle
\end{array}\right]
$$

$$
H\left(e_{3}\right)=\left[\begin{array}{ccccc}
\langle[0,0],[0,0],[0,0]\rangle & \langle[0.1,0.2],[0.4,0.6],[0.6,0.8]\rangle & \langle[0,0],[0,0],[0,0]\rangle & \langle[0,0],[0,0],[0,0]\rangle & \langle[0.1,0.2],[0.5,0.06],[0.6,0.7]\rangle \\
\langle[0.1,0.2],[0.4,0.6],[0.6,0.8]\rangle & \langle[0,0],[0,0],[0,0]\rangle & \langle[0.3,0.4],[0.5,0.6],[0.7,0.8]\rangle & \langle[0.2,0.3],[0.4,0.5],[0.8,0.9]\rangle & \langle[0.1,0.2],[0.7,0.8],[0.8,0.9]\rangle \\
\langle[0,0],[0,0],[0,0]\rangle & \langle[0.3,0.4],[0.5,0.6],[0.7,0.8]\rangle & \langle[0,0],[0,0],[0,0]\rangle & \langle[0.1,0.2],[0.6,0.7],[0.6,0.8]\rangle & \langle[0,0],[0,0],[0,0]\rangle \\
\langle[0,0],[0,0],[0,0]\rangle & \langle[0.2,0.3],[0.4,0.5],[0.8,0.9]\rangle & \langle[0.1,0.2],[0.6,0.7],[0.6,0.8]\rangle & \langle[0,0],[0,0],[0,0]\rangle & \langle[0.1,0.2],[0.5,0.6],[0.7,0.8]\rangle \\
\langle[0.1,0.2],[0.5,0.6],[0.6,0.7]\rangle] & \langle[0.1,0.2],[0.7,0.8],[0.8,0.9]\rangle & \langle[0,0],[0,0],[0,0]\rangle & \langle[0.1,0.2],[0.5,0.6],[0.7,0.8]\rangle & \langle[0,0],[0,0],[0,0]\rangle
\end{array}\right]
$$

After performing parametric $\vee$ and parametric $\wedge$ operations we obtain two resultant interval-valued neutrosophic graph $H(e)$ and $H^{\prime}(e)$. The incidence matrices of $H(e)$ and $H^{\prime}(e)$ are given by as follows.

$$
H(e)=\left[\begin{array}{ccccc}
\langle[0,0],[0,0],[0,0]\rangle & \langle[0.1,0.2],[0.4,0.6],[0.6,0.8]\rangle & \langle[0,0],[0,0],[0,0]\rangle & \langle[0,0],[0,0],[0,0]\rangle & \langle[0.1,0.2],[0.5,0.6],[0.4,0.6]\rangle \\
\langle[0.1,0.2],[0.4,0.6],[0.6,0.8]\rangle & \langle[0,0],[0,0],[0,0]\rangle & \langle[0.3,0.4],[0.5,0.6],[0.5,0.6]\rangle & \langle[0.2,0.3],[0,0],[0,0]\rangle & \langle[0.3,0.4],[0.4,0.5],[0.5,0.8]\rangle \\
\langle[0,0],[0,0],[0,0]\rangle & \langle[0.3,0.04],[0.5,0.6],[0.5,0.6]\rangle & \langle[0,0],[0,0],[0,0]\rangle & \langle[0.2,0.3],[0.6,0.7],[0.2,0.4]\rangle & \langle[0.3,0.4],[0,0],[0,0]\rangle \\
\langle[0,0],[0,0],[0,0]\rangle & \langle[0.2,0.03],[0,0],[0,0]\rangle & \langle[0.2,0.3],[0.6,0.7],[0.2,0.4]\rangle & \langle[0,0],[0,0],[0,0]\rangle & \langle[0.1,0.2],[0,0],[0,0]\rangle \\
\langle[0.1,0.2],[0.5,0.6],[0.4,0.6]\rangle & \langle[0.3,0.4],[0.4,0.5],[0.5,0.8]\rangle & \langle[0.3,0.4],[0,0],[0,0]\rangle & \langle[0.1,0.2],[0,0],[0,0]\rangle & \langle[0,0],[0,0],[0,0]\rangle
\end{array}\right]
$$




$$
H^{\prime}(e)=\left[\begin{array}{ccccc}
\langle[0,0],[0,0],[0,0]\rangle & \langle[0.1,0.2],[0.6,0.8],[0.6,0.9]\rangle & \langle[0,0],[0,0],[0,0]\rangle & \langle[0,0],[0,0],[0,0]\rangle & \langle[0.1,0.2],[0.8,0.9],[0.8,0.9]\rangle \\
\langle[0.1,0.2],[0.6,0.8],[0.6,0.9]\rangle & \langle[0,0],[0,0],[0,0]\rangle & \langle[0.1,0.4],[0.8,0.9],[0.7,0.9]\rangle & \langle[0,0],[0.7,0.8],[0.8,0.9]\rangle & \langle[0.1,0.2],[0.8,0.9],[0.8,0.9]\rangle \\
\langle[0,0],[0,0],[0,0]\rangle & \langle[0.1,0.4],[0.8,0.9],[0.7,0.9]\rangle & \langle[0,0],[0,0],[0,0]\rangle & \langle[0.1,0.2],[0.7,0.8],[0.8,0.9]\rangle & \langle[0,0],[0.7,0.9],[0.5,0.6]\rangle \\
\langle[0,0],[0,0],[0,0]\rangle & \langle[0,0],[0.7,0.8],[0.8,0.9]\rangle & \langle[0.1,0.2],[0.7,0.8],[0.8,0.9]\rangle & \langle[0,0],[0,0],[0,0]\rangle & \langle[0,0],[0.5,0.6],[0.7,0.8]\rangle \\
\langle[0.1,0.2],[0.8,0.9],[0.8,0.9]\rangle & \langle[0.1,0.2],[0.8,0.9],[0.8,0.9]\rangle & \langle[0,0],[0.7,0.9],[0.5,0.6]\rangle & \langle[0,0],[0.5,0.6],[0.7,0.8]\rangle & \langle[0,0],[0,0],[0,0]\rangle
\end{array}\right]
$$

The average possible membership degree of an element $x$ in given interval-valued neutrosophic set

$$
A=\left\{\left\langle x,\left[\inf _{A}(x), \sup _{A}(x)\right],\left[\operatorname{infI}_{A}(x), \operatorname{supI}_{A}(x)\right],\left[\inf F_{A}(x), \sup F_{A}(x)\right]\right\rangle\right\}
$$

is calculated by

$$
\begin{aligned}
S(x) & =\frac{1}{3}\left[\frac{\inf T_{A}(x)+\operatorname{supT}_{A}(x)}{2}+1-\frac{\operatorname{infI} I_{A}(x)+\operatorname{supI} I_{A}}{2}+1-\frac{\inf F_{A}(x)+\sup F_{A}}{2}\right] \\
& =\frac{\inf T_{A}(x)+\sup T_{A}(x)+4-i n f I_{A}(x)-\operatorname{supI} I_{A}(x)-\inf F_{A}(x)-\operatorname{supF} F_{A}(x)}{6}
\end{aligned}
$$

Based on $S_{k}$ we construct the tabular representation of score value of incidence matrix of resultant interval-valued neutrosophic graphs $H(e)$ and $H^{\prime}(e)$ with $S_{k}$ and choice value for each football club $t_{k}$ for $k=1,2,3,4,5$

Table 2: Tabular representation of score values with choice values of $H(e)$.

\begin{tabular}{|c|c|c|c|c|c|c|}
\hline & $t_{1}$ & $t_{2}$ & $t_{3}$ & $t_{4}$ & $t_{5}$ & $t^{\prime}{ }_{k}$ \\
\hline$t_{1}$ & 0.667 & 0.317 & 0.667 & 0.667 & 0.367 & 2.683 \\
\hline$t_{2}$ & 0.317 & 0.667 & 0.417 & 0.750 & 0.417 & 2.567 \\
\hline$t_{3}$ & 0.667 & 0.417 & 0.667 & 0.433 & 0.783 & 2.967 \\
\hline$t_{4}$ & 0.667 & 0.750 & 0.433 & 0.667 & 0.717 & 3.233 \\
\hline$t_{5}$ & 0.367 & 0.417 & 0.783 & 0.717 & 0.667 & 2.950 \\
\hline
\end{tabular}

Table 3: Tabular representation of score values with choice values of $H^{\prime}(e)$

\begin{tabular}{|c|c|c|c|c|c|c|}
\hline & $t_{1}$ & $t_{2}$ & $t_{3}$ & $t_{4}$ & $t_{5}$ & $t^{\prime \prime}{ }_{k}$ \\
\hline$t_{1}$ & 0.667 & 0.233 & 0.667 & 0.667 & 0.150 & 2.383 \\
\hline$t_{2}$ & 0.233 & 0.667 & 0.200 & 0.133 & 0.150 & 1.383 \\
\hline$t_{3}$ & 0.667 & 0.200 & 0.667 & 0.183 & 0.217 & 1.933 \\
\hline$t_{4}$ & 0.667 & 0.133 & 0.183 & 0.667 & 0.233 & 1.883 \\
\hline$t_{5}$ & 0.150 & 0.150 & 0.217 & 0.667 & 0.667 & 1.417 \\
\hline
\end{tabular}

After taking the arithmetic average of $t^{\prime}{ }_{k}$ and $t^{\prime \prime}{ }_{k}$ for all $k \in \Lambda=\{1,2,3,4,5\}$, we obtain the table shown in the below. 
Table 4: Tabular representation of average score values of $H(e)$ and $H^{\prime}(e)$.

\begin{tabular}{|c|c|c|c|}
\hline & $t^{\prime}{ }_{k}$ & $t^{\prime \prime}{ }_{k}$ & $t_{k}$ \\
\hline$t_{1}$ & 2.383 & 2.683 & 2.533 \\
\hline$t_{2}$ & 1.383 & 2.567 & 1.975 \\
\hline$t_{3}$ & 1.933 & 2.967 & 2.450 \\
\hline$t_{4}$ & 1.833 & 3.233 & 2.558 \\
\hline$t_{5}$ & 1.417 & 2.950 & 2.183 \\
\hline
\end{tabular}

Clearly, the maximum score value is 2.558 , scored by the $t_{4}$. Then the investor should purchase the football club $t_{4}$ which is the best choice for him.

Now we present our approach as an algorithm that is used in our application to deal with a decision-making problem.

\section{Algorithm}

(i) Input the set $P$ of choice of parameters of an investor or a businessman, $A$ is subset of $P$.

(ii) Input the interval-valued neutrosophic soft sets $(K, A)$ and $(M, A)$.

(iii) Construct the interval-valued neutrosophic soft graph $\tilde{G}=\left(G^{*}, K, M, A\right)$.

(iv) Compute the resultant interval-valued neutrosophic graphs $H(e)$ and $H^{\prime}(e)$ with

$$
\begin{aligned}
& H(e)=\underset{k \in \Lambda}{\tilde{V}} H\left(e_{k}\right) \text { for } e=\underset{k \in \Lambda}{\vee} e_{k}, \text { for all } k \in \Lambda . \\
& H^{\prime}(e)=\underset{k \in \Lambda}{\tilde{\Lambda}} H\left(e_{k}\right) \text { for } e=\bigwedge_{k \in \Lambda} e_{k}, \text { for all } k \in \Lambda .
\end{aligned}
$$

(v) Consider the interval-valued neutrosophic graph $H(e)$ and $H^{\prime}(e)$, and construct theirs incidence matrix forms.

(vi) Calculate the score $S_{k}$ of $t_{k}$ for all $k \in \Lambda$.

(vii) The decision is $t_{k}$ if $t_{k}^{\prime}=\max t_{k}$.

\section{Conclusion}

Graph theory is an extremely useful mathematical tool to solve the complicated problems in different fields. The intervalvalued neutrosophic soft models give more sensitive, flexibility and conformity to the systems as compared to the intervalvalued fuzzy soft models. We have applied the notion of interval-valued neutrosophic soft graph in a decision making problem. Also, a case study has been taken to exhibit the simplicity of the technique. Future work in this regard would be required to study whether the notions put forward in this paper yield a fruitful result.

\section{Competing interests}

The authors declare that they have no competing interests.

\section{Authors' contributions}

All authors have contributed to all parts of the article. All authors read and approved the final manuscript. 


\section{References}

[1] F. Smarandache, Neutrosophic set-a generalization of the intuitionistic fuzzy set. Granular Computing, 2006 IEEE International Conference, (2006) 38-42.

[2] H .Wang, Y. Zhang and R. Sunderraman, Truth-value based interval neutrosophic sets. Granular Computing, 2005 IEEE International Conference, 1 (2005) 274-277.

[3] A. Q. Ansari, R. Biswas and S. Aggarwal, Neutrosophic classifier: An extension of fuzzy classifier. Applied Soft Computing, 13 (2013) 563-573.

[4] H. Zhang, J. Wang and X. Chen, An outranking approach for multi-criteria decision-making problems with interval-valued neutrosophic sets. Neural Computing and Applications, 27(3) (2015) 1-13.

[5] H.Y. Zhang, P. Ji, J. Q. Wang and X. HChen, An Improved Weighted Correlation Coefficient Based on Integrated Weight for Interval Neutrosophic Sets and its Application in Multi-criteria Decisionmaking Problems. International Journal of Computational Intelligence Systems, 8(6) (2015).

[6] I. Deli, M. Ali and F. Smarandache, Bipolar neutrosophic sets and their application based on multicriteria decision making problems, Advanced Mechatronic Systems (ICAMechS), International Conference, (2015) 249-254.

[7] M. Ali and F. Smarandache, Complex Nuetrosophic Set. Neural Computing and Applications, 27(1) (2016) 1-18.

[8] M. Ali, I. Deli and F. Smarandache, The Theory of Neutrosophic Cubic Sets and Their Applications in Pattern Recognition. Journal of Intelligent and Fuzzy Systems, 30(4) 2016 1957-1963.

[9] D. Molodtsov, Soft set theory-first results, Computers and Mathematics with Applications, 37 (1999) 19-31.

[10] P.K. Maji, Neutrosophic soft set. Annals of Fuzzy Mathematics and Informatics, 5(1) (2013) 157-168.

[11] I. Deli, Interval-valued neutrosophic soft sets and its decision making, International Journal of Machine Learning and Cybernetics, 8 (2015) 1-12.

[12] L. Euler, Solutio problematis ad geometriam situs pertinentis, Commentarii Academiae Scientiarum Imperialis Petropolitanae, 8 (1736) $128-140$.

[13] S.Broumi, M. Talea, A.Bakali and F. Smarandache, Interval Valued Neutrosophic Graphs, Critical review, 12 (2016) 5-33.

[14] Y. Çelik and G. Kara, Combination of interval-valued neutrosophic soft sets and graph theory, New Trends in Mathematical Sciences, 6(2) (2018) 84-96. 